Cinémas

Revue d'études cinématographiques

Journal of Film Studies

\title{
L'industrie cinématographique allemande après la guerre... froide : année zéro, deuxième prise
}

\section{Monica Haim}

Volume 7, numéro 1-2, automne 1996

URI : https://id.erudit.org/iderudit/1000941ar

DOI : https://doi.org/10.7202/1000941ar

Aller au sommaire du numéro

Éditeur(s)

Cinémas

ISSN

1181-6945 (imprimé)

1705-6500 (numérique)

Découvrir la revue

Citer cet article

Haim, M. (1996). L'industrie cinématographique allemande après la guerre... froide : année zéro, deuxième prise. Cinémas, 7(1-2), 225-235.

https://doi.org/10.7202/1000941ar
Résumé de l'article

Cet article tente de faire état de la situation actuelle du cinéma allemand en Allemagne. Pour ce faire, il explore trois filons : la condition périphérique de ce cinéma sur son propre territoire; la conception allemande de l'auteur et de son cinéma; la refonte des conditions de production au lendemain de la réunification de l'Allemagne. 


\title{
L'industrie cinématographique allemande après la guerre... froide : année zéro, deuxième prise ${ }^{1}$
}

\section{Monica Haim}

\begin{abstract}
RÉSUMÉ
Cet article tente de faire état de la situation actuelle du cinéma allemand en Allemagne. Pour ce faire, il explore trois filons: la condition périphérique de ce cinéma sur son propre territoire; la conception allemande de l'auteur et de son cinéma; la refonte des conditions de production au lendemain de la réunification de l'Allemagne.
\end{abstract}

\section{ABSTRACT}

This article seeks to account for the current situation of German cinema in Germany. Towards this end it explores three veins: the peripherical condition of this cinema on its own territory; the German conception of the author and his/her cinema; the remodelling of the conditions of production on the morrow of German reunification.

L'Allemagne ne connaît ni diminution du nombre de salles (3400 écrans), ni baisse de leur fréquentation, ni décroissance des investissements d'État dans la production des films (220 millions de $\mathrm{DM}^{2}$ ). Toutefois, il y sévit une crise: celle de l'occupation des écrans. Les films allemands sont tassés dans un coin. Ils n'en occupent que $10 \%$ contre $80 \%$ pour les films états-uniens.

À l'exception des exploitants de salle dont le commerce se porte très bien, cet état des choses trouble profondément l'ensemble du milieu cinématographique. On envie, par exem- 
ple, au cinéma francais son taux de $30 \%$ d'occupation des écrans en France.

Il faut savoir, cependant, que depuis la Deuxième Guerre mondiale, le cinéma allemand n'a jamais occupé plus de $40 \%$ de l'espace des écrans nationaux (Elsaesser, p. 17). La situation ne résulte donc pas d'une invasion récente. Elle serait, selon les gens du milieu, le résultat d'une lente dérive des publics.

Ainsi, ne trouvant plus qu'un auditoire économiquement insignifiant, les exploitants de salles se détournent de la production locale: au pire, ils la refusent; au mieux, ils lui concèdent des périodes peu avantageuses ${ }^{3}$.

Qu'y a-t-il donc de "pourri” dans le monde du cinéma allemand? Il est facile d'imaginer que lorsqu'ils cherchent à répondre à cette question, les cinéastes, les producteurs, les fonctionnaires responsables du cinéma, les distributeurs et les critiques ont à l'esprit le fait que l'Allemagne est le plus peuplé ( 80 millions d'habitants), le plus riche pays d'Europe, la troisième puissance économique du monde et, que, mis à part le succès commercial de ses voitures, de ses appareils électroménagers, de son industrie chimique, pharmaceutique, etc., son cinéma (le «Nouveau Cinéma allemand") a joui, il y a à peine une dizaine d'années, d'un immense prestige mondial. Par contraste, l'impuissance actuelle des films allemands à reconquérir un modeste $10 \%$ à $15 \%$ de leur marché intérieur doit leur paraître d'autant plus pathétique.

Mais il ne faut pas oublier que le prestige du Nouveau Cinéma allemand était considérablement plus grand à l'étranger qu'en Allemagne même et que, au-dedans comme au-dehors, son attrait se limitait à un public relativement confidentiel ${ }^{4}$. Il faut aussi rappeler l'immense travail effectué par le Gœthe Institut à travers le monde pour favoriser l'essor de ce cinéma et savoir qu'en Allemagne, au même moment, sa distribution en salle était faiblement soutenue, bien plus faiblement que la production. Ce déséquilibre, qui se poursuit à l'heure actuelle, a engendré, dit-on, un "cinéma orphelin". Il importe cependant de souligner que le sens de ce qualificatif devient compliqué et ambigu si l'on considère le rôle capital tenu par la télévision dans la coproduction et surtout dans la diffusion de ces films ${ }^{5}$. 


\section{Le Nouveau Cinéma allemand était un cinéma d'auteur}

En Allemagne, la légitimation sociale de l' "auteur" est celle d'un producteur de culture. Et c'est en tant que tel qu'il est subventionné par l'État ${ }^{6}$. Ce dernier ne perçoit pas essentiellement l'auteur comme un bénéficiaire de ses largesses, mais plutôt comme une sorte de "métayer" auquel il loue une partie de ses revenus fiscaux en échange de légitimation et de prestige culturels ${ }^{7}$.

Sa pratique est pensée non comme une "politique" ou une esthétique, mais comme une "économie" ou une éthique. Suivant un modèle artisanal, il prend en charge toutes les étapes décisives de la fabrication du film (scénario, production et réalisation) et de sa "mise-en-société ".

De surcroît, pour ce type d'auteur né de l'esprit progressiste et antiautoritaire de 68, la notion de marché, de consommateurs et de transactions basées sur la valeur d'échange n'existe pas. Le théâtre de ses actions, c'est la société; leur destinataire, c'est la collectivité et la base des transactions, c'est la valeur d'usage.

Mais l'auteur est plus qu'un artiste engagé. Il ne se contente pas d'observer son temps et de façonner un objet qui le dénoncerait à distance; qui, de plus, par sa vente à un grossiste (un distributeur) puis à un détaillant (un exploitant de salle) se tranformerait en marchandise. Tout au contraire, les auteurs présentaient leurs films dans un réseau de salles parallèles ${ }^{8}$; ils les accompagnaient et ils les soumettaient au public comme matériau de réflexion et sujet de débat.

La mise sur pied d'un réseau alternatif de salles, tout en créant un débouché pour les films d'auteur, avait aussi pour effet de fidéliser un public important (majoritairement constitué d'étudiants). De plus, la présentation du film par son auteur et la discussion qui s'ensuivait (très animée et parfois interminable) créaient une proximité et instauraient un climat de complicité entre destinateur et destinataire?. (Dans ces conditions, les films étaient très loin de l'état d'orphelin.) Cette complicité, toutefois, n'était pas le résultat de la seule coprésence dans les salles de l'auteur et de son public. Elle procédait d'une détresse commune, d'une communauté d'aspirations, d'une cohésion idéologique et 
d'un sentiment d'urgence. De ce fait, les discussions à propos de ces films très proches de la réalité ambiante portaient davantage sur leur fond idéologique, sur la vision du monde de l'auteur que, par exemple, sur la structure narrative du récit et autres considérations esthétiques. Si le public cherchait dans les films des idées sociopolitiques justes et des analyses cohérentes aux dépens du plaisir esthétique et de la culture cinématographique, c'est qu'il cherchait à saisir et à comprendre sa société: son lourd passé et son présent contradictoire.

En faisant abstraction des caractères et des moyens spécifiques au média, en semblant ignorer que le fond idéologique d'un film se trouve davantage dans son "art" que dans son analyse, cette orientation discursive de la réception des films construit l'auteur en maître à penser. Mais cette construction ne résulte pas seulement de la réception. Elle est aussi le fait de l'artiste qui assume sa responsabilité sociale, qui utilise son média d'expression et sa légitimation sociale pour intervenir dans les tiraillements de son temps au nom de la vérité et de la justice et, ainsi, devient un intellectuel.

L'exigence première du public envers le Nouveau Cinéma allemand était une exigence intellectuelle. Cette exigence, bien qu'entièrement partagée par les auteurs, ne pouvait être satisfaite avec un égal succès par chacun.

\section{Le virage ${ }^{10}$}

Aujourd'hui, 33 ans après le manifeste d'Oberhausen qui jetait les fondations du Nouveau Cinéma allemand, ce dernier se résume, à l'étranger comme en Allemagne, aux trois auteurs (Fassbinder, Herzog, Wenders) dont l'œuvre est, artistiquement, la plus aboutie. Et, tandis qu'à l'étranger le phénomène du Nouveau Cinéma allemand se constitue en objet d'étude et en souvenir d'autant plus agréable que la déception est grande face aux films actuels ", en Allemagne il représente surtout un échec à surmonter, en ceci qu'il serait, selon certains, à la source de la désaffection du public allemand vis-à-vis de son propre cinéma et donc de sa défection.

"Déjà au milieu des années soixante-dix, dit Ula Stökl, nos films ne marchaient plus faute de public." Ceci a entraîné, bien 


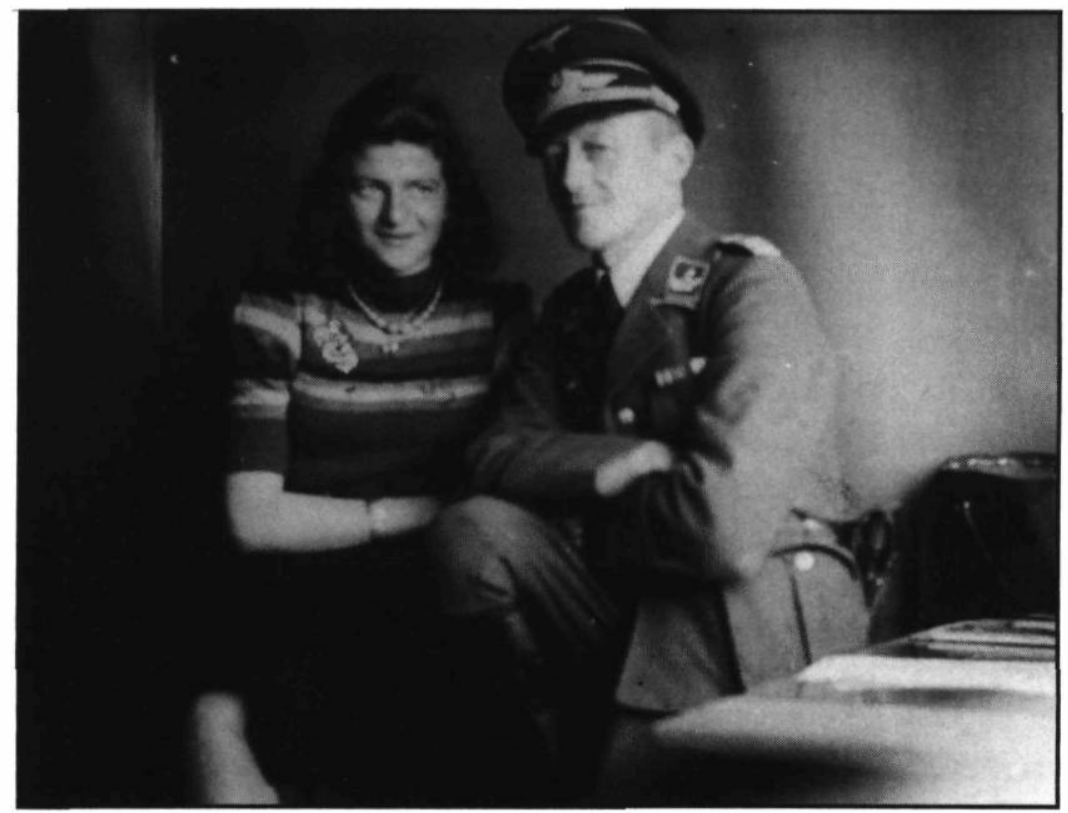

\section{Die Jüdin und der Hauptmann de Ulf von Mechow (1995)}

sûr, le démantèlement progressif du réseau parallèle. Les salles ont fermé ou elles ont orienté leur programmation vers des films garantissant une plus grande popularité. Et, tandis que Helma Sanders-Brahms ne cesse de répéter que ses films sont davantage reconnus en France et au Japon qu'en Allemagne, Thomas Elsaesser fait remarquer que Edgar Reitz a dû attendre 25 ans avant de rencontrer un succès public, dans son propre pays, grâce à Heimat (1980-1984) (Elsaesser, p. 309). Pour compléter le bilan de l'échec du cinéma allemand sur son propre territoire, on cite Fassbinder dont seul Le Mariage de Maria Braun (1978) a triomphé auprès des spectateurs; on relève le fait que Le Tambour (1978-79) de Volker Schlöndorff n'a recueilli que 16\% de ses recettes en Allemagne malgré la palme d'or de Cannes et l'oscar du film étranger. Enfin, on dresse la liste des cinéastes partis réaliser ailleurs (ex. : Wolfgang Peterson et Wim Wenders).

Les raisons abondent pour expliquer la désaffection du public. Pour ceux qui veillent au commerce du cinéma, la cause 
principale semble être la mentalité des auteurs. Choyés par un régime de subventions indifférent aux problèmes d'exploitation, élevés au rang de maîtres absolus par le système de production artisanal, les cinéastes, disent-ils, ne réalisent pas pour un public, mais pour eux-mêmes et pour un cercle restreint d'amis. Ils réalisent pour exprimer leurs états d'âme sans s'interroger sur les désirs du public ${ }^{12}$. Ils leur reprochent donc d'obéir à une morale d'artiste d'avant-garde mais surtout d'avoir créé des films que le public juge graves, lourds, compliqués, arides et lugubres; trop lents et trop longs: bref, trop exigeants, trop ennuyeux, trop déprimants. En somme, ils auraient imprimé une mauvaise image du cinéma allemand dans l'esprit de leurs concitoyens ${ }^{13}$.

Dans une prespective plus analytique, Reinhard Hauff, aujourd'hui directeur le l'Académie allemande du cinéma et de la télévision de Berlin, explique la faiblesse actuelle du cinéma allemand et la force du cinéma états-unien comme suit :

Le Nouveau Cinéma allemand et ses films étaient, pour ses auteurs, une question de vie. Ce cinéma était né de la même pression, de la même passion qui avait poussé ses auteurs à l'engagement et à l'élaboration d'un projet d'opposition face à la société. Les films, donc, étaient l'expression concrète, à la fois, de cette opposition sociale et de l'opposition, corollaire, au langage conventionnel du cinéma exprimée par la destruction de ses formes (ex. : Alexander Kluge).

Aujourd'hui, constate-t-il, les idéologies sont en crise. Les oppositions qui existaient jadis au sein de la société ont été démantelées. Elles ont été résolues non par confrontation, par une explosion qui aurait donné un gagnant et un perdant, mais par un effondrement, une implosion qui a brouillé les repères. Dans le conflit qui a opposé l'Ouest à l'Est, par exemple, il n'est plus du tout clair, vu d'Allemagne, qui est gagnant et qui est perdant.

Or, poursuit-il, ce que l'on recherche dans l'art, c'est une clarté que l'on ne retrouve plus dans la vie. Aux États Unis, les balises sont toujours claires: la culture en est une de l'action, de la lutte pour l'existence, un combat à la vie, à la mort où il n'y a pas de terrain neutre où survivre. De quoi $\mathrm{y}$ va-t-il dans les films 
américains sinon d'une lutte existentielle, d'un bottom line qui distingue très nettement le gagnant du perdant, le bon du méchant?

En Allemagne, le démantèlement des oppositions fait que les conflits sociaux ne peuvent pas être ou ne sont pas abordés. De ce fait, les histoires que nous racontons doivent fabriquer artificiellement ces oppositions. Elles doivent surgir de conflits pour être percutantes, car la notion de conflit implique la notion de résolution et donc le jaillissement d'une clarté. En d'autres termes, elles doivent pouvoir transposer ou sublimer les réels conflits.

Mais l'Allemagne n'a pas de grande tradition de conteurs. La sienne est surtout réflexive et spéculative. C'est une tradition d'essayistes. De plus, l'opposition entre la culture savante et la culture populaire est très marquée. Entre Gœthe et le photo-roman, il y a peu de place.

Ici, nous réfléchissons, nous cherchons des solutions et nous tournons en rond. Certes, les rues regorgent d'histoires, de conflits et de drames. Mais les récits qu'on en fait sont mus par une urgence autostimulée et minés par la conscience mélancolique de leur futilité. L'implosion des oppositions et des conflits a entrainé celle des modes même de la narration et de son possible impact ${ }^{14}$.

À cette analyse de l'incapacité actuelle des cinéastes de trouver une manière percutante de raconter, s'ajoute celle plus radicale de Ottokar Runze, ancien acteur du Berliner Ensemble, réalisateur et, aujourd'hui, producteur. Selon Runze, les subventions qui devaient protéger le cinéma de l'invasion états-unienne - elles ont cru, en effet, au rythme même de l'invasion - ont fini par le tuer. Pour étayer son avis, il cite les exemples de Fassbinder et de Herzog qui ont réalisé leur œuvre en dehors du système de subvention. En outre, il perçoit le cinéma d'auteur, au mieux, comme une cure d'amaigrissement (ex.: Antonioni); au pire, comme une hypocrisie de l'artiste (hypocrisie, parce qu'il se pense plus important que l'histoire qu'il raconte). Mais surtout, il le perçoit comme un cul-de-sac dont on ne peut sortir qu'en mettant sur l'écran des mises en scène inaccessibles à la télévision, de grands drames, des sujets qui touchent le cœur des spectateurs. 
Plusieurs font aussi état du faible niveau général de culture cinématographique du public et de la minceur du support critique dont dispose le cinéma. Il n'existe pas, en Allemagne, de revues de cinéma de niveau moyen, et bon nombre de journaux locaux ont aboli leur chronique de cinéma, jugeant que le grand volume publicitaire (des films états-uniens) rendait tout texte critique, en soi, inutile. De plus, l'accueil que les critiques réservent aux films de leur pays est tiède sinon pire: "La vie est trop courte pour se payer un film allemand", pour reprendre la citation de Veronika Rall, critique au Frankfurter Allgemeine Zeitung ${ }^{15}$.

\section{Tout est à refaire}

Installé à Potsdam-Babelsberg sur le terrain des studios de la DEFA et de la UFA avant elle, le Filmboard Berlin-Brandenburg $\mathrm{GmbH}$, fondé en 1994 à l'initiative des Länder de Berlin et de Brandebourg, est une créature de l'unification. Incorporé comme société à responsibilité limitée, il est, pour l'Allemagne, non seulement un nouveau type d'organisme d'aide au cinéma et l'annonciateur de l'avenir mais aussi, sur le plan symbolique, le signifiant du retour à l'ordre et aux sources de la grande industrie cinématographique d'avant-guerre.

Sa mission est de renverser le régime du cinéma d'auteur et de refondre la production sur des bases industrielles qui, on l'espère, favoriseront le retour du public.

D'entrée de jeu, le Filmboard GmbH differe, doublement, des autres organismes: premièrement parce qu'il est constitué en société commerciale - son capital ( 40 millions DM) doit être récupéré et non dépensé comme un budget de subventions - et deuxièmement parce que les décisions y sont prises par une seule personne (le directeur général) et non par un jury de pairs.

À ces différences fondamentales s'ajoute l'exigence que tout projet soit soumis par un "vrai" producteur qui doit assumer $30 \%$ du financement du budget et fournir une structure de récupération; qu'il soit accompagné d'un contrat de distribution et d'une identification claire du marché visé.

Il abolit, en outre, le "principe de l'arrosoir" (comme disent les Allemands) qui sous-tend l'actuelle répartition des subven- 
tions, pour accorder à un nombre restreint de projets une aide plus importante, des soins plus attentifs.

N'agissant pas en banquier mais en partenaire, son directeur général veut s'assurer que seuls entreront en production des projets absolument aboutis à tous égards. Par ailleurs, il entend se prévaloir de toutes les techniques de recherche qui permettent d'ajuster les films aux goûts et aux désirs des consommateurs, ainsi que de toutes les stratégies de mise en marché susceptibles de les attirer vers les produits.

En outre, dans un effort en vue de "professionnaliser" la profession, le Filmboard $\mathrm{GmbH}$ soutient l'organisation d'un grand nombre d'ateliers de scénarisation, de production, de mise en marché, etc., animés, principalement par des experts étatsuniens.

Vues d'ici, les politiques de la refonte n'ont rien d'exceptionnel. Mais en Allemagne, la normalisation du processus de production représente une véritable révolution (ou contre-révolution). Dans le contexte actuel, une culture de production aussi exceptionnelle que celle de l'Allemagne était vouée à la disparition. Néanmoins, bien des cinéastes qui avaient épousé la manière et la pensée "auteuriste" se sentent désorientés par son balayage et menacés par l'installation de la nouvelle donne.

Car, dorénavant, en Allemagne, comme de plus en plus à travers le monde, chacun veillera à l'accomplissement de sa tâche: les scénaristes scénariseront, les producteurs produiront, les réalisateurs réaliseront et les vendeurs vendront. Et, l'artisanat qu'on a voulu réhabiliter dans la ferveur politique des années soixante et soixante-dix cédera, encore une fois, la place à l'industrie. Ayant retrouvé la foi en la division du travail, la nouvelle génération de cinéastes et les entrepreneurs en cinéma espèrent qu'elle favorisera une union harmonieuse entre marché et création, art et commerce, qui restaurera la confiance des publics, chacun y trouvant le film de ses moyens et de ses exigences. À l'instar de l'industrie de l'automobile, dirais-je, celle du cinéma désire, elle aussi, fabriquer ses Volkswagen, ses Mercedes et ses Porsche.

Chercheure autonome 


\section{NOTES}

1 Je souhaite remercier les personnes suivantes pour la chaleur de leur accueil et l'intérêt de leurs propos: Mathias Allary (réalisateur), Heinz Badewitz (Festival de Berlin: Nouveaux Films allemands), Marianne Bergmann (Vertrieb Kontor, Hambourg), Clara Burckner (Basis-Film, Verleih), Bruno Fischli (Gœthe Institut, Munich), Lily Grote (réalisatrice), Reinhard Hauff (réalisateur, directeur de l'Académie de cinéma et de télévision de Berlin), Theo Hinz (Filmverlag der Autoren), Peter Kärte (critique, Frankfurter Rundschau), Prof. Klaus Keil (Filmboard Berlin-Brandebourg GmbH), Elfi Mikesch (camerawoman, réalisatrice), Susanne Reinker (ExportUnion des deutschen Films), Erika Richter (la revue Film und Fernseh), Ottokar Runze (producteur), Wolfram Skovronnek-Schaer (Cinepool), Ute Schneider (EFDO), Ula Stökl (réalisatrice), Regina Ziegler (productrice).

Je souhaite remercier aussi le Gœthe Institut de Montréal, dont le soutien matériel a permis cette enquête menée à Berlin en février 1995 et, particulièrement, Brigitte Hubmann.

2 Le taux de fréquentation a augmenté, d'ailleurs, de $25 \%$ en 1993.

3 Cette situation affecte les distributeurs allemands qui, en général, disposent de minces budgets publicitaires. Par contre, un film allemand distribué par un des majors ne subit pas ce sort. Le dernier film de Doris Dörrie, Qui máaime?, par exemple, est distribué par Buena Vista. Le seul budget pour la publicité télévisuelle fut de 1.5 million de DM ce qui, pour un distributeur allemand, représente bien plus que l'ensemble de son budget maximum.

4 Aujourd'hui encore, les distributeurs exportent plus facilement leurs films qu'ils ne trouvent, en Allemagne, de salle pour les présenter.

5 Les changements des politiques de la télévision d'État en matière de coproduction et de diffusion entraînés par l'avènement des stations privées et des cablodistributeurs ne seront pas discutés dans cet article pour des raisons pratiques. Ils devraient faire l'objet d'une étude particulière.

6 Le système de subvention distingue formellement et institutionnellement les films à vocation culturelle qui, au niveau fédéral, relèvent du ministère de l'Intérieur et les films à vocation commerciale qui, au même niveau, relèvent de la Filmförderunganstalt. Les subventions accordées aux premiers sont financées par les revenus fiscaux tandis que celles accordées aux seconds sont financées par une taxe perçue sur chaque billet vendu. Cette double structure est reprise au niveau des Länder. Parallèlement, le Gœethe Institut se charge de la diffusion des films culturels à l'étranger alors que la Export-Union s'occupe de la promotion du cinéma commercial.

7 Rappelons pour mémoire que, d'une part, après la guerre, l'Allemagne avait un énorme travail de reconstruction d'image culturelle à faire et que, d'autre part, sa division en deux États adversaires a fait que l'Allemagne fédérale a voulu s'imposer comme seul État légitime.

8 Ces salles qui s'appelaient "salles communales" étaient subventionnées par les revenus fiscaux des villes à l'instar des théâtres et des maisons d'opéra.

9 Cette tradition du débat se poursuit aujourd'hui lors du festival de Berlin dans le cadre du Forum du jeune cinéma. L'observateur étranger est toujours étonné par le niveau et l'intensité des discussions et souvent surpris par l'animosité qui s'y exprime.

10 En allemand, "die Wende». C'est le terme utilisé en Allemagne pour signifier l'unification.

11 Je voudrais, toutefois, signaler trois films documentaires remarquables réalisés cette année: Kalte-Heimat de Volker Koepp, LaBENDIG de Hannes Schönemann (ces deux réalisateurs proviennent de l'ex-Allemagne de l'Est) et Die Jüdin und der Hauptmann de Ulf von Mechow. 
12 La structure du système de subventions est aujourd'hui contestée par l'ensemble de la profession parce qu'elle est trop lourde, trop complexe et, surtout, parce qu'elle éparpille l'argent à travers le pays.

13 Certains nuancent ce jugement en attribuant cette mauvaise image à une cause plus profonde: la mauvaise conscience des Allemands et leur haine de soi.

14 Entretien avec l'auteure, Berlin, le 7 février 1995.

15 "Visage dans la foule: Fate de Fred Kelemen, au Festival du film de Toronto", Frankfurter Allgemeine Zeitung (28 septembre 1994). Rall cite cette phrase lapidaire pour désavouer l'attitude de ses collègues.

\section{OUVRAGE CITÉ}

Elsaesser, Thomas. New German Cinema: A History. New Brunswick (New Jersey): Rutgers University Press, 1989. 